\title{
Co-delivery of doxorubicin and pH-sensitive curcumin prodrug by transferrin-targeted nanoparticles for breast cancer treatment
}

\author{
TONGXING CUI ${ }^{1}$, SIHAO ZHANG ${ }^{1}$ and HONG SUN ${ }^{2}$ \\ ${ }^{1}$ Department of Galactophore Surgery and ${ }^{2}$ Second Department of Oncology, \\ Qingdao Municipal Hospital, Qingdao, Shandong 266011, P.R. China
}

Received July 13, 2016; Accepted December 5, 2016

DOI: $10.3892 /$ or.2017.5345

\begin{abstract}
The natural product curcumin and the chemotherapeutic agent doxorubicin have been used in the treatment of many cancers, including breast cancer. However, fast clearance and unspecific distribution in the body after intravenous injection are still challenges to be overcome by an ideal nanosized drug delivery system in cancer treatment. In this study we design transferrin (Tf) decorated nanoparticles (NPs) to co-deliver CUR and DOX for breast cancer treatment. A pH-sensitive prodrug, transferrin-poly(ethylene glycol)curcumin (Tf-PEG-CUR), was synthesized and used for the self-assembling of NPs (Tf-PEG-CUR NPs). DOX is incorporated into the Tf-PEG-CUR NPs to obtain Tf-PEG-CUR/DOX NPs. In vitro cytotoxicity studies and in vivo antitumor activity were carried out using MCF-7 cells and mice bearing MCF-7 cells, respectively. Tf-PEG-CUR/DOX NPs has a particle size of $89 \mathrm{~nm}$ and a zeta potential of $-15.6 \mathrm{mV}$. This system displayed remarkably higher efficiency than other systems both in vitro and in vivo. DOX and CUR were successfully loaded into nanocarriers. The in vitro cell viability assays revealed the combination of Tf-PEG-CUR and DOX NPs exhibited higher cytotoxicity in vitro in MCF-7 cells compared with Tf-PEG-CUR NPs alone. Using the breast cancer xenograft mouse model, we demonstrate that this co-encapsulation approach resulted in an efficient tumor-targeted drug delivery, decreased cytotoxic effects and exhibited stronger antitumor effect.
\end{abstract}

\section{Introduction}

Combination chemotherapy has long been adopted as the standard of care against various cancer types. Compared to

Correspondence to: Dr Hong Sun, Second Department of Oncology, Qingdao Municipal Hospital, Qingdao, Shandong 266011, P.R. China E-mail: sunh_qdmh@163.com

Key words: co-delivery, breast cancer, hydrazone bond, transferrin, doxorubicin, curcumin prodrug the single-drug chemotherapy, the proper drug combination, especially the combination of cytotoxic and chemosensitizing agents exhibited better synergistic actions and target selectivity, more effective for tumor localization, thus overcoming multi-drug resistance (MDR) (1-3). For instance, the combination of anticancer agents such doxorubicin (DOX) and curcumin (CUR) has been shown to modulate different signaling pathways in breast cancer cells, which are beneficial to reverse multidrug resistance (MDR) $(4,5)$.

Curcumin (CUR), a naturally occurring polyphenol extracted from the rhizome Curcuma longa, is widely used in China and India as a traditional herb (6). Recently, more and more studies have shown that CUR, either alone or in combination with other anticancer agents, has pleiothropic antineoplastic effects, due to modulation of nuclear factorkappa $\mathrm{B}(\mathrm{NF}-\kappa \mathrm{B})$ and other cell signaling pathways (7-9). Furthermore, CUR is known to inhibit the ATP-binding cassette (ABC) drug transporters implicated in cancer MDR, such as $\mathrm{P}$-glycoprotein (P-gp), breast cancer resistance protein (ABCG2/BCRP), and multidrug resistance-associated proteins 1 and 5 (MRP1 and MRP5) (10-12). Therefore, CUR may be the optimum small-molecule chemosensitizer and brings new hope for the combination therapy of breast cancer. However, the clinical application of CUR is still hindered due to its low aqueous solubility, extreme degradation and metabolization which are responsible for poor bioavalability and pharmacological activity (13). Hence, it is highly desirable to develop a novel drug delivery system to solve these obstacles.

Stimuli-responsive and prodrug-based nanoassemblies have many advantages as a potent platform for co-delivery of anticancer drugs, such as improved drug availability, and high drug loading efficiency, to realize responsive release of large amount of drugs (14). In this study, a novel $\mathrm{pH}$-sensitive CUR prodrug was designed. It was composed of poly(ethylene glycol)-aldehyde (PEG-CHO), adipodihydrazide (ADH), and CUR by the $\mathrm{pH}$-sensitive biodegradable chemical linkers (hydrazone bonds). Furthermore, dual targeted nanoparticles (NPs), pH-sensitive and transferrin (Tf) functionalized NPs, were engineered based on the prodrug technology.

Among the various types of ligands used for active targeting, transferrin (Tf) or folate (FA) stands out to be a desirable choice for malignant tumors, since most solid tumor cells 
express a high level of $\mathrm{Tf}$ receptor (TfR) or folate receptor (FR) on their surfaces while the level of TfR or FR is much lower in non-epithelial tumors and normal tissues (15-18). Tf and FA, both have been extensively used to decorate PEG-based amphiphilic nanomaterials for the development of drug delivery system (18). Our group has constructed folate (FA) decorated nanostructured lipid carriers (NLCs) for targeted delivery of curcumin (CUR). The results demonstrated that FA-CUR-NLCs were efficient in selective delivery to MCF-7 human breast cancer cells overexpressing FA receptors (FRs) (19). In this study, we designed Tf decorated nanoparticles (NPs) to co-deliver CUR and DOX for breast cancer treatment.

The $\mathrm{pH}$-sensitive prodrug, Tf-PEG-CUR, was synthesized. Tf-PEG-CUR was capable of self-assembling into nanoparticles (Tf-PEG-CUR NPs). Because of the hydrophobic nature of DOX, it could be entrapped within the Tf-PEG-CUR NPs in the core of the NPs to obtain Tf-PEG-CUR/DOX NPs. In vitro cytotoxicity studies and in vivo antitumor activity were carried out using MCF-7 cells and mice bearing MCF-7 cells, respectively. This system was anticipated to achieve stable, dual targeting NPs of CUR and DOX, to improve synergistic anticancer effects, and reduce toxicity.

\section{Materials and methods}

Materials. Doxorubicin (DOX), curcumin (CUR), human Tf (iron-free), maleic anhydride (MA), 1-ethyl-3-(3-dimethylamino-propyl) carbodiimide (EDC) and adipic acid dihydrazide (ADH) were purchased from Sigma-Aldrich (St. Louis, MO, USA). CHO-PEG-CHO (average molecular weight, $3.4 \mathrm{kDa}$ ) was purchased from Taiyuan Pegchem Technology Co., Ltd. (Shanxi, China). N,N'-dicyclohexylcarbodimide (DCC) and 4-dimethylaminopyridine (DMAP) were obtained from GL Biochem Co., Ltd. (Shanghai, China). Doxorubicin hydrochloride liposome injection (DOX Lip) and doxorubicin hydrochloride injection (DOX Inj) were purchased from Cardinal Health China (Shanghai, China). All other chemicals were of analytical grade or higher.

Cells and animals. The MCF-7 human breast cancer cell lines (MCF-7 cells) were obtained from American Type Culture Collection (Manassas, VA, USA) and cultured in Dulbecco's Modified Eagle's medium (DMEM) (Sigma, St. Louis, MO, USA) supplemented with $10 \%$ fetal bovine serum (FBS) (Fisher Chemicals, Fairlawn, NJ, USA) and $100 \mathrm{U} / \mathrm{ml}$ penicillin and $100 \mathrm{mg} / \mathrm{ml}$ streptomycin (Sigma) in a $5 \% \mathrm{CO}_{2}$ fully humidified atmosphere. All experiments were performed on cells in the logarithmic phase of growth.

BALB/c nude mice (6-8 weeks) were purchased from the Vital River Laboratory Animal Technology Co. Ltd. (Beijing, China). Mice were acclimated at $25^{\circ} \mathrm{C}$ and $55 \%$ of humidity under natural light/dark conditions for 7 days before the experiment. All the animal experiments were performed in accordance with the Animal Management Rules of the Ministry of Health of the People's Republic of China.

Synthesis and characterization ofTf-PEG-CUR.Tf-PEG-CUR was achieved by four-step reaction (Fig. 1). Firstly, CUR (0.2 mmol), MA (0.15 mmol), DCC (0.2 mmol), DMAP (0.02 mmol) were dissolved in anhydrous dimethyl sulfoxide
(DMSO). The reaction mixture was stirred at $400 \mathrm{rpm}$ for $24 \mathrm{~h}$ under the protection of nitrogen, and filtered to remove byproducts. The solution was poured into $10 \mathrm{ml}$ cold ethyl acetate. The solvent was removed by a rotary evaporator to obtain CUR-MA.

Secondly, CUR-MA (0.1 mmol), ADH $(0.2 \mathrm{mmol})$ and EDC $(0.2 \mathrm{mmol})$ were dissolved in DMSO. The reaction mixture was stirred at $400 \mathrm{rpm}$ for $24 \mathrm{~h}$ under the protection of nitrogen. CUR-ADH was obtained by vacuum drying.

Thirdly, Tf-PEG-CHO was synthesized. CHO-PEG-CHO and Tf were dissolved in acetate buffer ( $\mathrm{pH} 5.0$ ), then $20 \mathrm{mM}$ sodium cyanoborohydride $\left(\mathrm{NaCNBH}_{3}\right)$ was added under stirring for $24 \mathrm{~h}$ (20). The reaction mixture was separated by gel-filtration chromatography.

Finally, CUR-ADH was conjugated to Tf-PEG-CHO via a hydrazone linkage formed between the ketone group of Tf-PEG-CHO and the hydrazide end group of CUR-ADH. Tf-PEG-CHO (0.05 mmol) and CUR-ADH (0.05 mmol) were dissolved in DMSO, then $3 \mu \mathrm{l}$ triethylamine was added and reacted under stirring for $24 \mathrm{~h}$ at room temperature. The sample was purified by dialysis with a dialysis bag and freeze-dried to obtain Tf-PEG-CUR. Chemical structure of Tf-PEG-CUR was analyzed by ${ }^{1} \mathrm{H}-\mathrm{NMR}$ spectroscopy.

Preparation of Tf-PEG-CUR NPs and Tf-PEG-CUR/DOX NPs. Tf-PEG-CUR (100 mg) was dissolved in DMSO $(5 \mathrm{ml})$ at $25^{\circ} \mathrm{C}$, and then the solution was dropwise added to $10 \mathrm{ml}$ of PBS under gentle stirring $(21,22)$. The solutions were dialyzed against excess PBS with a dialysis bag (MWCO: $10 \mathrm{kDa}$ ) for $48 \mathrm{~h}$, and then filtered through a $0.45-\mu \mathrm{m}$ pore-sized microporous membrane to obtain Tf-PEG-CUR NPs. Tf-PEG-CUR/ DOX NPs (Fig. 2) were prepared with the same procedure as Tf-PEG-CUR NPs by adding DOX into the DMSO solution: the Tf-PEG-CUR (100 mg) and DOX (10 mg) were dissolved in DMSO $(5 \mathrm{ml})$ at $25^{\circ} \mathrm{C}$. CUR and DOX mixed injection (CUR/DOX Inj) was prepared by adding $10 \mathrm{mg}$ of CUR into $5 \mathrm{ml}$ of DOX Inj (5 ml : $10 \mathrm{mg}$ ). The obtained NPs and Inj were stored at $2-8^{\circ} \mathrm{C}$.

Particle morphology, size and zeta potential. Particle morphology of Tf-PEG-CUR/DOX NPs was observed by a transmission electron microscopy (TEM, Hitachi, Tokyo, Japan) (23). Diluted NPs were placed on a carbon-coated copper grid, negatively stained with $2 \%$ phosphotungstic acid, and then observed with TEM. The size, polydispersity index, and zeta potential of Tf-PEG-CUR NPs and Tf-PEG-CUR/ DOX NPs were measured using a Zetasizer (Nano ZS 90, Malvern, Worcestershire, UK).

Drug encapsulation efficiency and drug loading capacity. The encapsulation efficacy (EE) and drug loading capacity (DL) of DOX and CUR was determined using ultrafiltration method. The amount of DOX was determined with F-4500 fluorescence spectrophotometer (emission wavelength: $480 \mathrm{~nm}$, excitation wavelength: $556 \mathrm{~nm}$, Hitachi). The amount of CUR was measured with an HPLC method at $420 \mathrm{~nm}$ using Agilent 1260 Infinity LC (Agilent Technologies, Santa Clara, CA, USA). HPLC analyses were performed on a Hypersil ODS2 C18 column ( $250 \mathrm{~mm} \times 4.6 \mathrm{~mm}, 5 \mu \mathrm{m})$. The mobile phase used was acetonitrile: $4 \%$ glacial acetic acid in water (v/v, 45:55). The 
<smiles>[R]C([R])=O</smiles><smiles>O=C(O)/C=C/C(=O)OCCl</smiles>

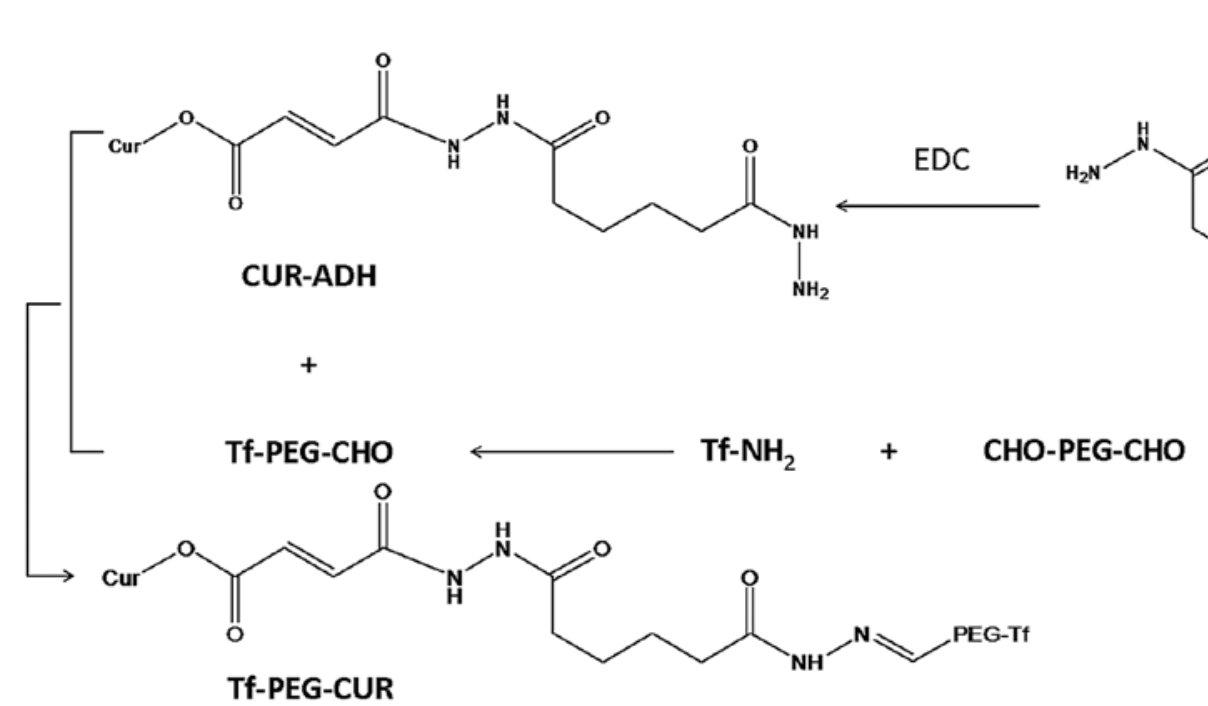

$+$

Figure 1. Synthesis scheme of Tf-PEG-CUR.

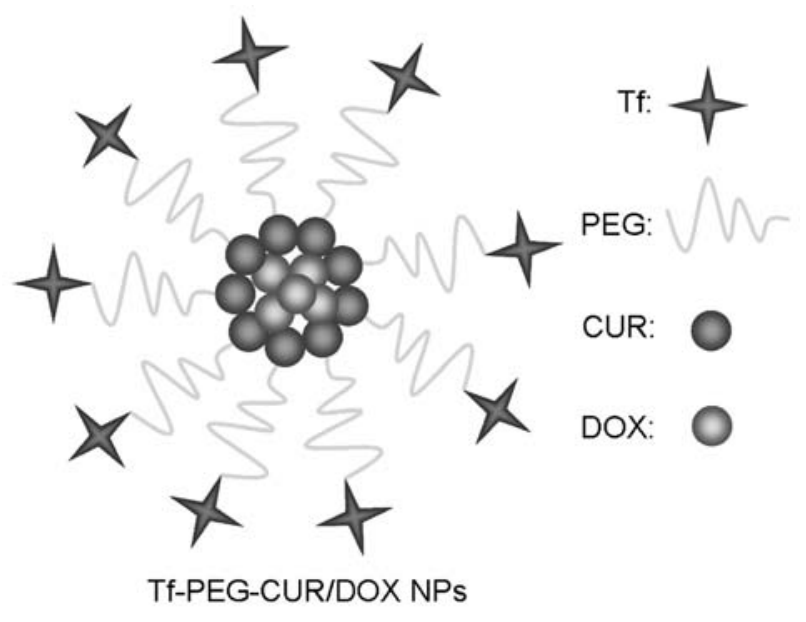

Figure 2. Schematic diagram of Tf-PEG-CUR/DOX NPs.

EE and DL were calculated using the following equations (6): $\mathrm{EE}(\%)=$ the amount of loaded DOX or CUR / total amount of DOX or CUR used for NP preparation $x 100$. DL $(\%)=$ the amount of loaded DOX or CUR / total amount of the DOX or CUR and NPs x 100.

In vitro stability in plasma. Plasma stability of Tf-PEG-CUR/ DOX NPs was evaluated (24). Tf-PEG-CUR/DOX NPs were incubated in phosphate buffer (PBS) solution containing $10 \%$ fetal bovine serum (FBS, v/v) at $37^{\circ} \mathrm{C}$ for $24 \mathrm{~h}$, respectively. At scheduled times (0, 1, 2, 4, 8, 12 and $24 \mathrm{~h}), 1 \mathrm{ml}$ of each sample was diluted with $2 \mathrm{ml} \mathrm{THF}$ and the mixture was bath sonicated for $5 \mathrm{~min}$, followed by centrifugation at
$10,000 \mathrm{rpm}$ for $5 \mathrm{~min}$. The variation trends of the size and EE were calculated by the method described in the above two sections.

In vitro drug release study. CUR and DOX released from Tf-PEG-CUR/DOX NPs in vitro was investigated by the dialysis method against the release medium at $\mathrm{pH} 7.4$, or 5.0, respectively (25). Briefly, $2 \mathrm{ml}$ of NPs suspension was added into a dialysis tube with MWCO $3 \mathrm{kDa}$, and incubated in $40 \mathrm{ml}$ of release medium with then centrifuging at $100 \mathrm{rpm}$, at $37^{\circ} \mathrm{C}$. At different time-points, release medium $(2 \mathrm{ml})$ was collected and the same volume of fresh release medium was added into the system. CUR contents in the release medium were determined by the same method as described in 'Drug encapsulation efficiency and drug loading capacity'.

In vitro cytotoxicity assay. The cytotoxicity of Tf-PEG-CUR NPs and Tf-PEG-CUR/DOX NPs was determined using the standard MTT cell proliferation kit (ATCC) according to the manufacturer's protocol (26). DOX Lip, CUR/DOX Inj, and DOX Inj were used as contrast. Briefly, MCF-7 cells were seeded onto 96 -well plates with a density of $1 \times 10^{4}$ cells per well and incubated at $37^{\circ} \mathrm{C}$ in a humidified atmosphere of $95 \%$ air and $5 \% \mathrm{CO}_{2}$ for $16 \mathrm{~h}$. The medium in each well was replaced with $200 \mathrm{ml}$ of culture medium containing the samples and cultured for $72 \mathrm{~h}$. The medium in each well was then replaced with fresh medium and the cells were incubated for another $24 \mathrm{~h}$. The incubation medium was then replaced with $100 \mathrm{ml}$ of fresh medium and $10 \mathrm{ml}$ of MTT reagent. After $6 \mathrm{~h}, 100 \mathrm{ml}$ of detergent reagent was added to each well and incubated for $18 \mathrm{~h}$ at room temperature in the dark until all the 
Table I. Physicochemical characterization.

\begin{tabular}{lccccccc}
\hline Formulation & $\begin{array}{c}\text { Particle } \\
\text { size }(\mathrm{nm})\end{array}$ & $\begin{array}{c}\text { Polydispersity } \\
\text { index }\end{array}$ & $\begin{array}{c}\text { Zeta potential } \\
(\mathrm{mV})\end{array}$ & $\begin{array}{c}\text { CUR EE } \\
(\%)\end{array}$ & $\begin{array}{c}\text { DOX EE } \\
(\%)\end{array}$ & $\begin{array}{c}\text { CUR DL } \\
(\%)\end{array}$ & $\begin{array}{c}\text { DOX DL } \\
(\%)\end{array}$ \\
\hline Tf-PEG-CUR NPs & $89.5 \pm 3.4$ & $0.12 \pm 0.02$ & $-11.3 \pm 1.1$ & $84.6 \pm 2.8$ & N/A & $5.4 \pm 0.7$ & N/A \\
Tf-PEG-CUR/DOX NPs & $88.7 \pm 3.9$ & $0.14 \pm 0.03$ & $-15.6 \pm 1.6$ & $85.3 \pm 3.2$ & $82.7 \pm 4.1$ & $4.6 \pm 0.8$ & N/A \\
\hline
\end{tabular}

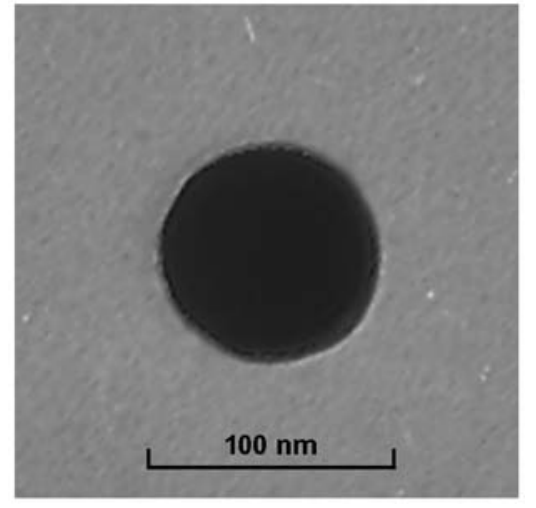

Figure 3. TEM image of Tf-PEG-CUR/DOX NPs.

crystals were dissolved. The absorbance intensity at $570 \mathrm{~nm}$ was recorded on a Bio-Rad Microplate Reader Model 550 (Bio-Rad Laboratories, Hercules, CA, USA). Cell viability was defined as the percent live cells compared with untreated controls. The half maximal inhibitory concentration $\left(\mathrm{IC}_{50}\right)$ values were calculated accordingly.

In vivo tissue distribution study. BALB/c mice were inoculated subcutaneously with $1 \times 10^{6} \mathrm{MCF}-7$ cells. MCF-7 tumor xenografts were grown in Balb/C mice and estrogen was provided as a $\beta$-estradiol pellet 1 week prior to the injection of the cells. The tumors were allowed to develop on the posterolateral side of the mice for one week prior to treatment to obtain the breast cancer-bearing animal model (27). Tf-PEG-CUR/DOX NPs and CUR/DOX Inj were injected into the mice by tail vein, respectively. In the in vivo organ distribution study each sample was investigated at $10 \mathrm{~min}, 1,8,24$ and $48 \mathrm{~h}$ after intravenous injection. At predetermined time intervals, mice were sacrificed and the tumor, heart, liver, spleen, lung and kidney were collected. Tissues were initially weighed and homogenized with physiological saline to determine the amount of DOX or CUR in each tissue. The concentrations of released DOX or CUR were determined as described in 'Drug encapsulation efficiency and drug loading capacity'.

In vivo antitumor evaluation. After the breast cancer-bearing animal model was built, the mice were randomized into 6 groups and intravenously injected once a week for 7 weeks with $0.9 \%$ normal saline (NS), Tf-PEG-CUR/DOX NPs, Tf-PEG-CUR NPs, DOX Lip, CUR/DOX Inj, and DOX Inj contained $50 \mathrm{mg} / \mathrm{kg}$ CUR and/or DOX $(4,28)$. Tumor growth was determined by measuring the major (L) and minor (W) diameter with a caliper. The tumor volume was calculated according to the formula: Tumor volume $=0.5 \times \mathrm{L} \mathrm{x} \mathrm{W}^{2}$. The
Table II. Changes in size and EE in the presence of serum.

\begin{tabular}{lccc}
\hline Time (h) & Particle size (nm) & CUR EE (\%) & DOX EE (\%) \\
\hline 0 & $88.9 \pm 4.1$ & $85.7 \pm 3.4$ & $82.9 \pm 3.8$ \\
1 & $89.1 \pm 3.6$ & $85.1 \pm 3.7$ & $82.3 \pm 3.4$ \\
2 & $89.8 \pm 4.3$ & $85.3 \pm 3.9$ & $82.8 \pm 3.7$ \\
4 & $90.6 \pm 3.9$ & $84.8 \pm 3.8$ & $82.1 \pm 3.5$ \\
8 & $89.4 \pm 4.6$ & $83.9 \pm 4.1$ & $81.6 \pm 4.0$ \\
12 & $91.1 \pm 4.5$ & $83.5 \pm 4.6$ & $80.9 \pm 4.4$ \\
24 & $90.7 \pm 3.8$ & $84.1 \pm 4.4$ & $81.3 \pm 4.2$ \\
\hline
\end{tabular}

body weight was measured. The inhibition rate of tumor growth (IRT) was calculated according to the following formula: IRT $(\%)=($ mean tumor weight of a control group - mean tumor weight of a treatment group) / mean tumor weight of a control group x 100 .

Statistical analysis. Assignment to treatments were made at random. Treatment comparisons were made by analysis of variance and protected least significant difference or Student's t-test. Differences were considered statistically significant at $\mathrm{P}<0.05$. Data are presented as means \pm standard errors.

\section{Results}

Characterization of Tf-PEG-CUR. As showed in Fig. 1, during the synthesis of Tf-PEG-CUR, hydrazone bond (-NH-N=CH-), amido linkage $(-\mathrm{NH}-\mathrm{C}=\mathrm{O})$, and ester bond $(-\mathrm{O}-\mathrm{C}=\mathrm{O})$ was formed. ${ }^{1} \mathrm{H}-\mathrm{NMR}$ analyses were performed to confirm the synthesis $(\delta, \mathrm{ppm}): 8.13$ (-NH-NH-); 7.59 (-N-N=CH-); 7.08 $(-\mathrm{NH}-\mathrm{N}=\mathrm{CH}-)$; 6.89 (-O-C=O-CH=); 6.57-6.79 ( $\mathrm{H}$ of benzene ring); $5.41\left(-\mathrm{CH}_{2}-\mathrm{O}-\mathrm{C}=\mathrm{O}\right)$. The proton signals in the ${ }^{1} \mathrm{H}-\mathrm{NMR}$ suggest the successful synthesis of Tf-PEG-CUR.

Characterization of Tf-PEG-CUR/DOX NPs. Tf-PEG-CUR/ DOX NPs display a spherical morphology with a smooth exterior (Fig. 3). The size of NPs measured was $\sim 90 \mathrm{~nm}$ and characterized by a relatively narrow size distribution (Table I). The zeta potential of Tf-PEG-CUR NPs and Tf-PEG-CUR/ DOX NPs was -11.3 and $-15.6 \mathrm{mV}$, respectively. CUR and DOX were efficiently loaded in Tf-PEG-CUR/DOX NPs, reaching the DL of 4.6 and $5.9 \%$ with the EE of 85.3 and $82.7 \%$, respectively.

In vitro plasma stability. Changes in size and EE in the presence of serum are described in Table II. Tf-PEG-CUR/DOX 


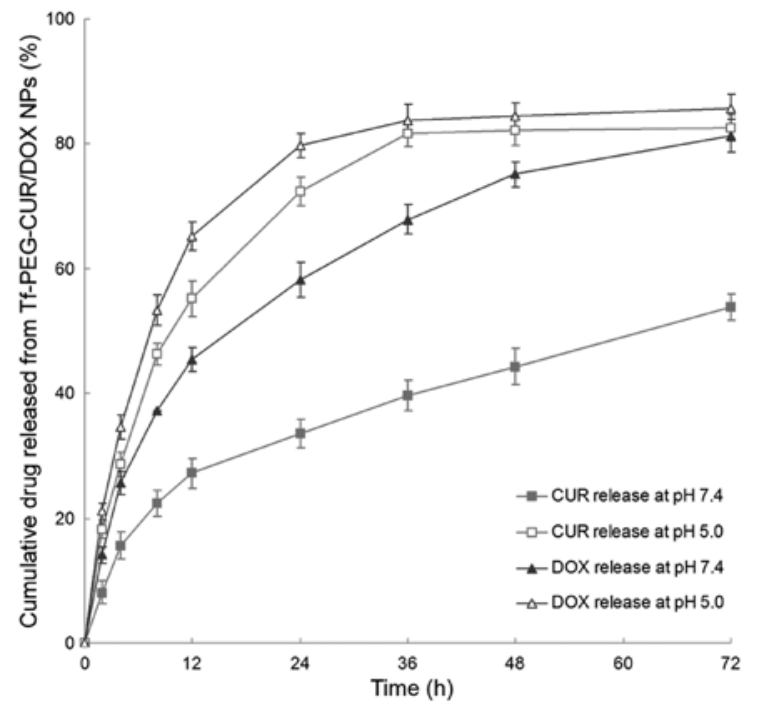

Figure 4. In vitro CUR and DOX release from Tf-PEG-CUR/DOX NPs.

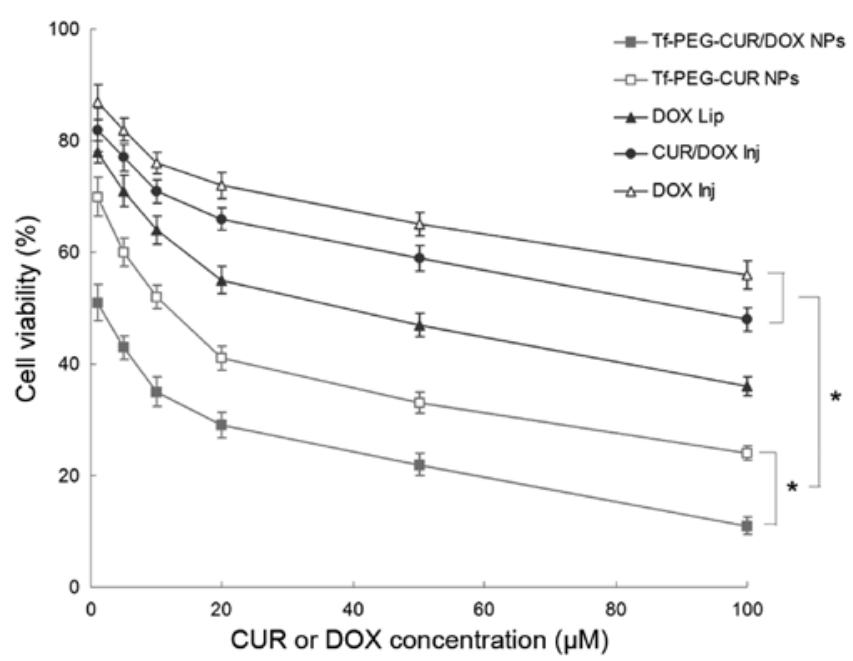

Figure 5. In vitro cytotoxicity results of NPs, liposomes and injections evaluated in MCF-7 cells ( $(\mathrm{P}<0.05)$.
NPs were stable up to $24 \mathrm{~h}$ without any significant size or EE changes. Thus, Tf-PEG-CUR/DOX NPs were considered very stable after incubation with FBS and suggest that this formulation will not aggregate or disassemble after intravenous administration.

Invitro drug release. The in vitro drug release from Tf-PEG-CUR/ DOX NPs was studied at $37^{\circ} \mathrm{C}$ under $\mathrm{pH} 7.4$ or 5.0. The results showed that CUR release was significantly accelerated under mildly acidic environments (Fig. 4). For example, 72.4 and $33.6 \%$ of CUR was released from NPs in $24 \mathrm{~h}$ at $\mathrm{pH} 5.0$ and 7.4 , respectively. Moreover, DOX release was also observed to be faster at $\mathrm{pH} 5.0$.

In vitro cytotoxicity. As shown in Fig. 5, Tf-PEG-CUR/DOX NPs exhibited relatively higher toxicity in comparison with Tf-PEG-CUR NPs in MCF-7 cells $(\mathrm{P}<0.05)$. NP formulations showed slightly higher toxicity than liposomes and injections $(\mathrm{P}<0.05)$. Table III summarizes the $\mathrm{IC}_{50}$ of different samples tested. The $\mathrm{IC}_{50}$ of Tf-PEG-CUR/DOX NPs was the lowest $(2.5 \mu \mathrm{M})$, which is many-fold dose advantage over the liposome and free drug injections.

In vivo tissue distribution. In vivo CUR and DOX tissue distribution outcomes of Tf-PEG-CUR/DOX NPs and CUR/ DOX Inj are shown in Figs. 6 and 7, respectively. The CUR and DOX concentration in tumor, lung and liver following injection of Tf-PEG-CUR/DOX NPs was higher than the injection of CUR/DOX Inj, whereas, the drug concentration of Tf-PEG-CUR/DOX NP group in heart and kidney was lower than CUR/DOX Inj group. Drug concentrations of Tf-PEG-CUR/DOX NPs group in the tumor tissue remained relatively stable at all time-points until $48 \mathrm{~h}$ after injection.

In vivo antitumor efficiency. In vivo antitumor activity of Tf-PEG-CUR/DOX NPs was performed in MCF-7/ADR tumor bearing mice. As shown in Fig. 8, tumor volumes of saline group increased markedly over time, while tumor volume remained almost unchanged in Tf-PEG-CUR/DOX

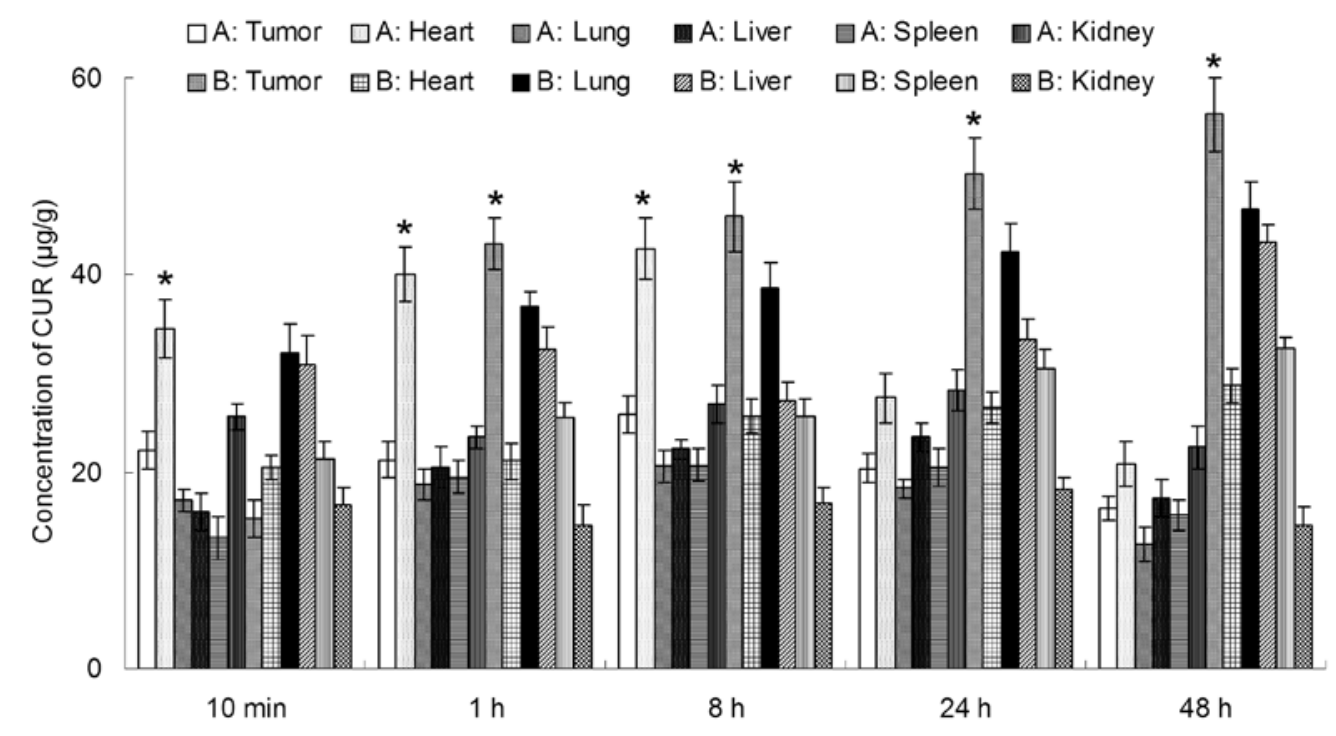

Figure 6. In vivo CUR tissue distribution outcomes of CUR/DOX Inj (A) and Tf-PEG-CUR/DOX NPs (B); ${ }^{*} \mathrm{P}<0.05$. 
Table III. $\mathrm{IC}_{50}$ values of in vitro cytotoxicity assay.

\begin{tabular}{lccccc}
\hline Formulation & Tf-PEG-CUR/DOX NPs & Tf-PEG-CUR NPs & DOX Lip & CUR/DOX Inj & DOX Inj \\
\hline IC $_{50}$ of CUR $(\mu \mathrm{M})$ & $2.6 \pm 0.3$ & $11.3 \pm 1.1$ & N/A & $83.5 \pm 5.6$ & N/A \\
IC $_{50}$ of DOX $(\mu \mathrm{M})$ & $2.6 \pm 0.3$ & N/A & $39.4 \pm 2.7$ & $83.5 \pm 5.6$ & $163.5 \pm 12.7$ \\
\hline
\end{tabular}

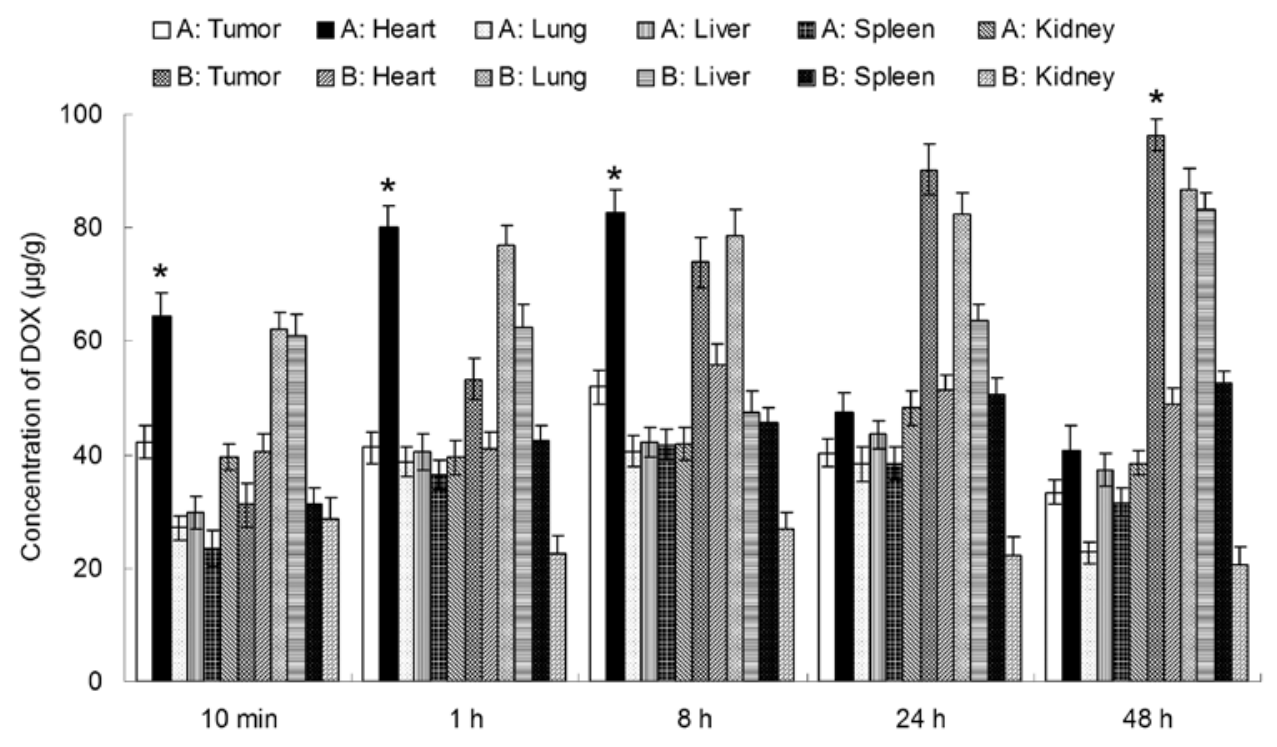

Figure 7. In vivo DOX tissue distribution outcomes of CUR/DOX Inj (A) and Tf-PEG-CUR/DOX NPs (B); ${ }^{*} \mathrm{P}<0.05$.

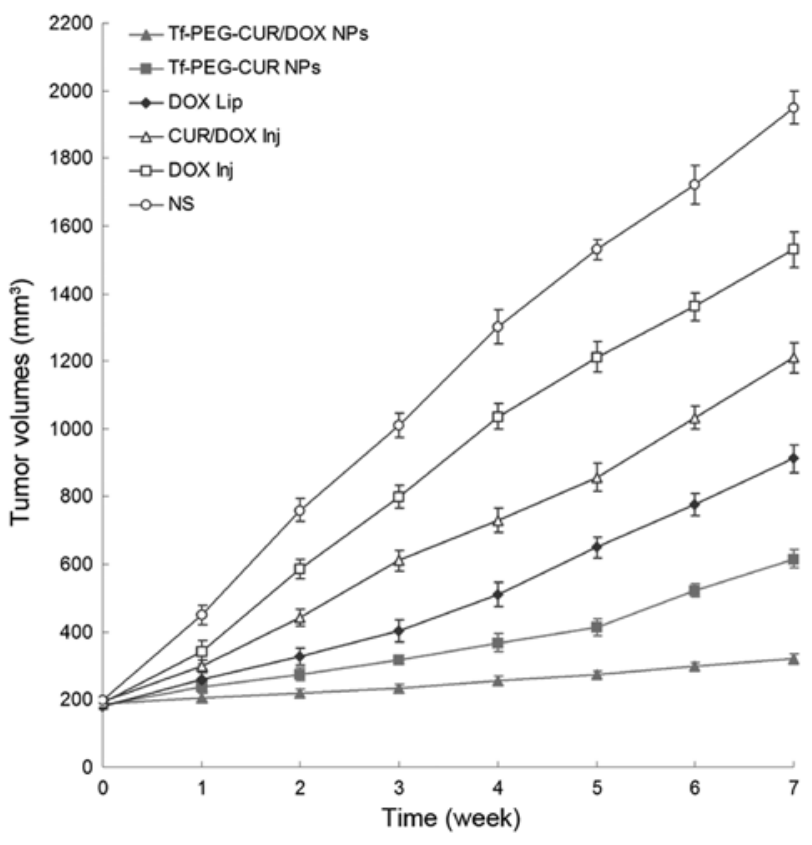

Figure 8. In vivo antitumor activity: tumor volumes of NPs, liposomes and injections evaluated in MCF-7/ADR tumor-bearing mice.

NP treated group. In contrast, other treatment groups showed a relatively weaker inhibitory effect on the tumor growth. Seven weeks after treatment, the tumors from each treatment group were weighed and IRT was calculated. The data of IRT in Fig. 9 show similar results to tumor volume. Tf-PEG-CUR/DOX

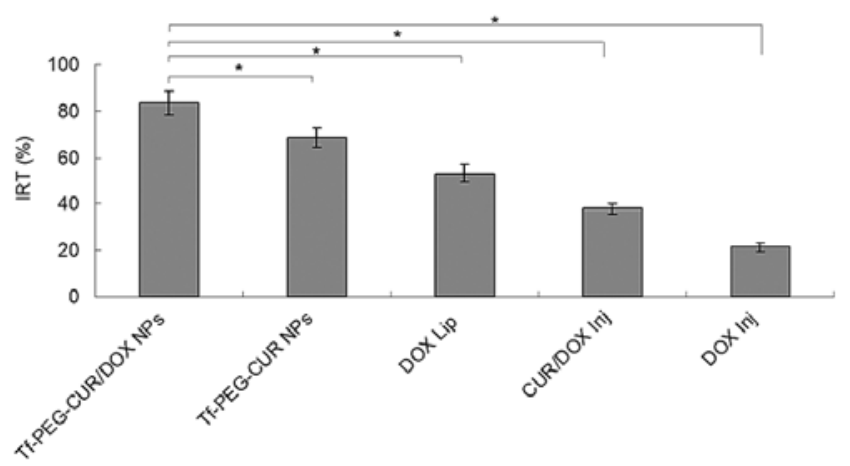

Figure 9. In vivo antitumor activity: IRT of NPs, liposomes and injections evaluated in MCF-7/ADR tumor-bearing mice (" $\mathrm{P}<0.05)$.

NPs had the highest IRT (83.5\%), while IRT of Tf-PEG-CUR NPs, DOX Lip, and CUR/DOX Inj were 68.4, 53.2 and 37.9\%, respectively. The obvious body weight loss could be observed in the free drug solution groups. However, NP groups did not cause significant body weight loss.

\section{Discussion}

This study developed and characterized the $\mathrm{pH}$-sensitive prodrug loaded self-assembled particles in order to evaluate them as a potential drug delivery system for the treatment of breast cancer. The novel system allows for effective simultaneous loading of lipophilic drugs. Such a strategy could be beneficial in treating many types of cancer that develops 
resistance to the same chemotherapeutic agent administered over time (29).

Tf-PEG-CUR/DOX NPs displayed beneficial physicochemical characteristics, including uniform particle size, narrow particle size distribution, high encapsulation efficacy and sustained drug release. Under TEM observation, Tf-PEG-CUR/DOX NPs exhibited a spherical morphology. The NPs showed a negatively charged surface and an average hydrodynamic diameter of $\sim 90 \mathrm{~nm}$ (Table I). The most likely origin of this negative charge is the presence of the anionic materials used in the preparation process. The PDI of each formulation was lower than 0.2 , indicating the homogeneous nature of the formulation (23). A successful NP system should have a good loading capacity and high encapsulation efficacy to reduce the quantity of the carrier required for administration (30). Tf-PEG-CUR/DOX NPs had EE of $>80 \%$ for both CUR and DOX, suggesting the fine loading efficiency of the NPs.

In vitro drug release study of Tf-PEG-CUR/DOX NPs was investigated at $\mathrm{pH}$ 7.4, and 5.0. In acidic media, the release of CUR was more rapid than that of the neutral environment. This may be because of the $\mathrm{pH}$-sensitive hydrazone bonds could cleave much more easily in low $\mathrm{pH}$ and release the drug faster (31). Besides, DOX release rate of also increased as $\mathrm{pH}$ value decreased from $\mathrm{pH} 7.4$ to 5.0. It could be explained by the degradation of the NPs that occurs predominantly via hydrazone bond cleavage at lower $\mathrm{pH}$ medium releasing the DOX loaded in the NPs (32). The $\mathrm{pH}$ value of the bloodstream is $\sim 7.4$, while the existing tumoral $\mathrm{pH}$ and that of endocytic compartments of the cells generally range from 4 to $6(33,34)$. This difference in $\mathrm{pH}$ value makes $\mathrm{pH}$-triggered drug release possible. Thus, the use of $\mathrm{pH}$-sensitive biodegradable polymeric materials as drug delivery carries has been considered as a common procedure. These properties can make the multifunctional drug loading NPs to internalize into tumor cells easier, and then release antitumor drug and kill the tumor cells.

The cytotoxicity data unambiguously showed that the pH-sensitive Tf-PEG-CUR/DOX NPs proved to significantly outclass the free drug in terms of relative potency (25). It inhibited the viability and proliferation of the breast cancer cell lines at low concentrations, with $\mathrm{IC}_{50}$ value many times lower than other contrast formula tested. Higher toxicity of Tf-PEG-CUR/DOX NPs in comparison with Tf-PEG-CUR NPs may be evidence of the co-delivery of the two drugs has a synergistic effect to inhibit cancer cells. $\mathrm{IC}_{50}$ values of NP formulations were much lower than liposome and free drug injections. This may because the $\mathrm{pH}$-sensitive prodrug containing NPs would disassemble after internalized by cancer cells, and the CUR and DOX released from NPs continuously accumulated within the tumor cells, and then killed cancer cells (22).

In vivo drug distribution in heart and kidney may cause systemic toxicity; on the contrary, distribution mainly in tumor tissue compared with the other tissues could decrease the side effects and lead to better antitumor therapeutic efficiency (35). Solid tumors have leakage in the micro-vasculature and the nano-sized particles could target the tumor owing to the enhanced permeability and retention (EPR) effects (36). EPR effects prevented the entry of nanoformulation in the normal cell at the same time favoring selective entry into tumors, which resulted in efficient drug accumulation in tumor tissue (37). Drug concentrations of Tf-PEG-CUR/DOX NP group in the tumor tissue remained high until $48 \mathrm{~h}$ after injection, indicating the sustained-release behavior of the DOX+CUR LPNs. The long circulating effect of NPs was due to the presence of PEG chain on the surface of particles, which provided stealth effect to the NPs (38).

In vivo antitumor effect of the Tf-PEG-CUR/DOX NPs was evaluated in MCF-7/ADR tumor xenograft model. Compared with CUR/DOX Inj, Tf-PEG-CUR/DOX NPs presented a remarkably higher inhibition effect towards tumor growth, which is consistent with their in vitro efficiency (4). After Tf-PEG-CUR/DOX NP was intravenously injected, they would accumulate into tumor because NPs preferentially extravasated from pores in tumor vessel walls (39). Moreover, Tf-PEG-CUR/DOX NP was able to release drugs more quickly upon their arrival on the acidic tumor area, resulting in higher CUR and DOX concentration in the local region of the tumor. This corresponded with the results of drug concentration in the tumor. Higher IRT $(83.5 \%)$ than that of DOX Lip (53.2\%) suggests better anticancer ability of NPs than their liposome counterparts. In order to evaluate the systemic toxicity of different systems, the body weight variation was calculated. No obvious body weight loss of the NP groups illustrated the low systemic toxicity of the systems.

In conclusion, we developed self-assembled NPs containing $\mathrm{pH}$-sensitive prodrug, which can be used as a nanocarrier for the co-delivery of hydrophobic anticancer drugs. The results demonstrate that co-encapsulation of CUR and DOX in NPs can promote the cytotoxicity by both drugs in vitro in breast cancer cells and in vivo in breast cancerbearing mouse model. The synergistic effect is important and may provide combinatorial strategies in cancer therapy. This study suggests that simultaneous delivery of CUR and DOX by Tf-PEG-CUR/DOX NPs might be a promising treatment for breast cancer.

\section{References}

1. Hu CM and Zhang L: Nanoparticle-based combination therapy toward overcoming drug resistance in cancer. Biochem Pharmacol 83: 1104-1111, 2012.

2. Huang P, Wang D, Su Y, Huang W, Zhou Y, Cui D, Zhu X and Yan D: Combination of small molecule prodrug and nanodrug delivery: Amphiphilic drug-drug conjugate for cancer therapy. J Am Chem Soc 136: 11748-11756, 2014.

3. Saraswathy M and Gong S: Different strategies to overcome multidrug resistance in cancer. Biotechnol Adv 31: 1397-1407, 2013.

4. Guo S, Lv L, Shen Y, Hu Z, He Q and Chen X: A nanoparticulate pre-chemosensitizer for efficacious chemotherapy of multidrug resistant breast cancer. Sci Rep 6: 21459, 2016.

5. Li WM, Chiang CS, Huang WC, Su CW, Chiang MY, Chen JY and Chen SY: Amifostine-conjugated $\mathrm{pH}$-sensitive calcium phosphate-covered magnetic-amphiphilic gelatin nanoparticles for controlled intracellular dual drug release for dual-targeting in HER-2-overexpressing breast cancer. J Control Release 220: 107-118, 2015.

6. Duan J, Mansour HM, Zhang Y, Deng X, Chen Y, Wang J, Pan Y and Zhao J: Reversion of multidrug resistance by co-encapsulation of doxorubicin and curcumin in chitosan/poly(butyl cyanoacrylate) nanoparticles. Int J Pharm 426: 193-201, 2012.

7. Sinha D, Biswas J, Sung B, Aggarwal BB and Bishayee A: Chemopreventive and chemotherapeutic potential of curcumin in breast cancer. Curr Drug Targets 13: 1799-1819, 2012. 
8. Mezzanotte L, An N, Mol IM, Löwik CW and Kaijzel EL: A new multicolor bioluminescence imaging platform to investigate $\mathrm{NF}-\kappa \mathrm{B}$ activity and apoptosis in human breast cancer cells. PLoS One 9: e85550, 2014.

9. Aggarwal BB, Shishodia S, Takada Y, Banerjee S, Newman RA, Bueso-Ramos CE and Price JE: Curcumin suppresses the paclitaxel-induced nuclear factor-kappaB pathway in breast cancer cells and inhibits lung metastasis of human breast cancer in nude mice. Clin Cancer Res 11: 7490-7498, 2005.

10. Angelini A, Iezzi M, Di Febbo C, Di Ilio C, Cuccurullo F and Porreca E: Reversal of P-glycoprotein-mediated multidrug resistance in human sarcoma MES-SA/Dx- 5 cells by nonsteroidal anti-inflammatory drugs. Oncol Rep 20: 731-735, 2008.

11. Revalde JL, Li Y, Hawkins BC, Rosengren RJ and Paxton JW: Heterocyclic cyclohexanone monocarbonyl analogs of curcumin can inhibit the activity of ATP-binding cassette transporters in cancer multidrug resistance. Biochem Pharmacol 93: 305-317, 2015.

12. Zhou Q, Ye M, Lu Y, Zhang H, Chen Q, Huang S and Su S: Curcumin improves the tumoricidal effect of mitomycin $\mathrm{C}$ by suppressing ABCG2 expression in stem cell-like breast cancer cells. PLoS One 10: e0136694, 2015.

13. Burgos-Morón E, Calderón-Montaño JM, Salvador J, Robles A and López-Lázaro M: The dark side of curcumin. Int J Cancer 126: $1771-1775,2010$

14. Cheng R, Meng F, Deng C, Klok HA and Zhong Z: Dual and multistimuli responsive polymeric nanoparticles for programmed site-specific drug delivery. Biomaterials 34: 3647-3657, 2013.

15. Qian ZM, Li H, Sun H and Ho K: Targeted drug delivery via the transferrin receptor-mediated endocytosis pathway. Pharmacol Rev 54: 561-587, 2002.

16. Widera A, Norouziyan F and Shen WC: Mechanisms of TfR-mediated transcytosis and sorting in epithelial cells and applications toward drug delivery. Adv Drug Deliv Rev 55: 1439-1466, 2003

17. Mulik RS, Mönkkönen J, Juvonen RO, Mahadik KR and Paradkar AR: Transferrin mediated solid lipid nanoparticles containing curcumin: Enhanced in vitro anticancer activity by induction of apoptosis. Int J Pharm 398: 190-203, 2010.

18. Chen S, Yang K, Tuguntaev RG, Mozhi A, Zhang J, Wang PC and Liang XJ: Targeting tumor microenvironment with PEG-based amphiphilic nanoparticles to overcome chemoresistance. Nanomedicine 12: 269-286, 2016.

19. Lin M, Teng L, Wang Y, Zhang J and Sun X: Curcumin-guided nanotherapy: A lipid-based nanomedicine for targeted drug delivery in breast cancer therapy. Drug Deliv 23: 1420-1425, 2016.

20. Shao Z, Shao J, Tan B, Guan S, Liu Z, Zhao Z, He F and Zhao J: Targeted lung cancer therapy: Preparation and optimization of transferrin-decorated nanostructured lipid carriers as novel nanomedicine for co-delivery of anticancer drugs and DNA. Int J Nanomed 10: 1223-1233, 2015.

21. Carlson LJ, Cote B, Alani AW and Rao DA: Polymeric micellar co-delivery of resveratrol and curcumin to mitigate in vitro doxorubicin-induced cardiotoxicity. J Pharm Sci 103: 2315-2322, 2014.

22. Zhang Y, Yang C, Wang W, Liu J, Liu Q, Huang F, Chu L, Gao H, Li C, Kong D, et al: Co-delivery of doxorubicin and curcumin by $\mathrm{pH}$-sensitive prodrug nanoparticle for combination therapy of cancer. Sci Rep 6: 21225, 2016.

23. Zhao X, Chen Q, Li Y, Tang H, Liu W and Yang X: Doxorubicin and curcumin co-delivery by lipid nanoparticles for enhanced treatment of diethylnitrosamine-induced hepatocellular carcinoma in mice. Eur J Pharm Biopharm 93: 27-36, 2015.

24. Qu J, Zhang L, Chen Z, Mao G, Gao Z, Lai X, Zhu X and Zhu J: Nanostructured lipid carriers, solid lipid nanoparticles, and polymeric nanoparticles: Which kind of drug delivery system is better for glioblastoma chemotherapy? Drug Deliv: 08 Jun, 2016 (Epub ahead of print)
25. Jelezova I,Drakalska E, Momekova D, Shalimova N, Momekov G, Konstantinov S, Rangelov S and Pispas S: Curcumin loaded $\mathrm{pH}$-sensitive hybrid lipid/block copolymer nanosized drug delivery systems. Eur J Pharm Sci 78: 67-78, 2015.

26. Tang H, Murphy CJ, Zhang B, Shen Y, Van Kirk EA, Murdoch WJ and Radosz M: Curcumin polymers as anticancer conjugates. Biomaterials 31: 7139-7149, 2010.

27. Ling G, Zhang T, Zhang P, Sun J and He Z: Nanostructured lipidcarrageenan hybrid carriers (NLCCs) for controlled delivery of mitoxantrone hydrochloride to enhance anticancer activity bypassing the BCRP-mediated efflux. Drug Dev Ind Pharm 42: 1351-1359, 2016.

28. Lin L, Hutzen B, Zuo M, Ball S, Deangelis S, Foust E, Pandit B, Ihnat MA, Shenoy SS, Kulp S, et al: Novel STAT3 phosphorylation inhibitors exhibit potent growth-suppressive activity in pancreatic and breast cancer cells. Cancer Res 70: 2445-2454, 2010.

29. Garbuzenko OB, Winkler J, Tomassone MS and Minko T: Biodegradable Janus nanoparticles for local pulmonary delivery of hydrophilic and hydrophobic molecules to the lungs. Langmuir 30: 12941-12949, 2014.

30. Misra R and Sahoo SK: Coformulation of doxorubicin and curcumin in poly(D,L-lactide-co-glycolide) nanoparticles suppresses the development of multidrug resistance in K562 cells. Mol Pharm 8: 852-866, 2011.

31. Gu Y, Zhong Y, Meng F, Cheng R, Deng C and Zhong Z: Acetallinked paclitaxel prodrug micellar nanoparticles as a versatile and potent platform for cancer therapy. Biomacromolecules 14: 2772-2780, 2013

32. Zhou L, Liang D, He X, Li J, Tan H, Li J, Fu Q and Gu Q: The degradation and biocompatibility of $\mathrm{pH}$-sensitive biodegradable polyurethanes for intracellular multifunctional antitumor drug delivery. Biomaterials 33: 2734-2745, 2012.

33. Bae Y, Jang WD, Nishiyama N, Fukushima S and Kataoka K: Multifunctional polymeric micelles with folate-mediated cancer cell targeting and $\mathrm{pH}$-triggered drug releasing properties for active intracellular drug delivery. Mol Biosyst 1: 242-250, 2005.

34. Lee ES, Gao Z, Kim D, Park K, Kwon IC and Bae YH: Super $\mathrm{pH}$-sensitive multifunctional polymeric micelle for tumor $\mathrm{pHe}$ specific TAT exposure and multidrug resistance. J Control Release 129: 228-236, 2008.

35. Yang Y, Li N, Nie Y, Sheng M, Yue D, Wang G, Tang JZ and $\mathrm{Gu} \mathrm{Z}$ : Folate-modified poly(malic acid) graft polymeric nanoparticles for targeted delivery of doxorubicin: Synthesis, characterization and folate receptor expressed cell specificity. J Biomed Nanotechnol 11: 1628-1639, 2015.

36. Kobayashi H, Watanabe R and Choyke PL: Improving conventional enhanced permeability and retention (EPR) effects; what is the appropriate target? Theranostics 4: 81-89, 2013.

37. Mei L, Fu L, Shi K, Zhang Q, Liu Y, Tang J, Gao H, Zhang Z and $\mathrm{He} \mathrm{Q}$ : Increased tumor targeted delivery using a multistage liposome system functionalized with RGD, TAT and cleavable PEG. Int J Pharm 468: 26-38, 2014.

38. Nilsson C, Østergaard J, Larsen SW, Larsen C, Urtti A and Yaghmur A: PEGylation of phytantriol-based lyotropic liquid crystalline particles - the effect of lipid composition, PEG chain length, and temperature on the internal nanostructure. Langmuir 30: 6398-6407, 2014.

39. Tang J, Zhang L, Gao H, Liu Y, Zhang Q, Ran R, Zhang Z and $\mathrm{He} \mathrm{Q}$ : Co-delivery of doxorubicin and P-gp inhibitor by a reduction-sensitive liposome to overcome multidrug resistance, enhance anti-tumor efficiency and reduce toxicity. Drug Deliv 23: $1130-1143,2016$ 